\title{
Imagerie sismique par corrélation de bruit ambiant : du laboratoire à l'échelle globale
}

Pierre Boué (pierre.boue@univ-grenoble-alpes) et Anne Paul (anne.paul@univ-grenoble-alpes) Institut des Sciences de la Terre (Univ. Grenoble Alpes, Univ. Savoie Mont Blanc, CNRS, IRD, IFSTTAR), 1381 rue de la Piscine, BP 53, 38041 Grenoble Cedex

L'énergie sismique libérée par

une source naturelle ou artificielle

est habituellement utilisée pour

éclairer la structure interne

de la Terre. Aujourd'hui,

de nouvelles méthodes permettent

de se passer de ces sources

conventionnelles via l'utilisation

du bruit sismique ambiant qui,

après corrélation de longs

enregistrements, transforme

virtuellement chaque récepteur

en source. Cette méthode

d'imagerie du sous-sol est

également répétable dans

le temps, donnant ainsi accès

à une multitude d'applications

pour la surveillance

des structures géologiques.

Cet article présente le principe

de l'interférométrie sismique

par corrélation du bruit ambiant

à travers différents exemples,

de l'échelle du laboratoire

à l'échelle globale.

\section{Introduction à l'imagerie sismique}

L'analyse des enregistrements du mouvement du sol à la suite d'un séisme ou du déclenchement d'une source artificielle est à l'origine d'une grande partie de nos connaissances sur la structure interne de la Terre. À l'échelle globale, les séismes de magnitude supérieure à $\sim 5,5$ sont utilisés pour déterminer les grandes structures de notre planète - croute, manteau et noyau - et leurs hétérogénéités. L'emploi de sources déclenchées par l'homme (explosion, camion vibreur, chute d'une masse...) permet d'étudier les structures les plus superficielles présentant des enjeux économiques et sociétaux autour, par exemple, des réservoirs d'hydrocarbures et géothermiques, des gisements miniers, de la caractérisation géotechnique du proche sous-sol. Dans un article récent [1], B. Romanowicz a discuté les grands principes et les enjeux de l'utilisation des ondes sismiques pour l'imagerie ou la modélisation de la Terre profonde.

L'imagerie sismique est principalement basée sur l'analyse des temps de parcours entre deux positions, ainsi que sur l'amplitude des ondes sismiques: mesure absolue entre source et récepteur, ou mesure relative entre deux récepteurs. Selon les objectifs de l'étude et les paramètres de la source, différents types d'ondes et bandes de fréquence sont utilisés. Dans le domaine pétrolier par exemple, l'imagerie des réservoirs d'hydrocarbures est très majoritairement basée sur l'analyse des ondes $\mathrm{P}$ (ondes de compression) entre 10 et $1000 \mathrm{~Hz}$. Pour la Terre profonde, les ondes $\mathrm{P}$ et $\mathrm{S}$ (cisaillement) sont employées conjointement et à plus basse fréquence (environ 0,1 à $10 \mathrm{~Hz}$ ). Les propriétés de dispersion (vitesse dépendant de la fréquence) des ondes de surface (Rayleigh, Love) sont très largement utilisées pour imager les structures de la croute terrestre et du manteau supérieur dans la bande de fréquence entre 0,01 et $1 \mathrm{~Hz}$. Enfin, à encore plus basse fréquence, les modes propres de la Terre permettent aussi de contraindre les grandes échelles jusqu'au noyau interne.

\section{Fonction de Green et corrélation}

Un signal sismique issu d'une source quelconque et enregistré par un récepteur distant, porte à la fois l'information relative à la source (énergie, durée, directivité, etc.) et celle sur le trajet parcouru par les ondes entre source et récepteur. Au premier ordre, seule l'information portant sur le trajet est utile pour imager la structure ; il est donc nécessaire de s'affranchir au maximum de l'information liée à la source. On appelle fonction de Green la fonction de transfert élastodynamique du milieu de propagation. C'est la réponse de la structure à une sollicitation ponctuelle en temps et en espace, qui décrit entièrement et de manière unique la propagation des ondes élastiques entre source et récepteur (fig. 1a). Plusieurs stratégies existent pour estimer la fonction de Green à partir de signaux sismiques, la plus rapide étant simplement de considérer la 


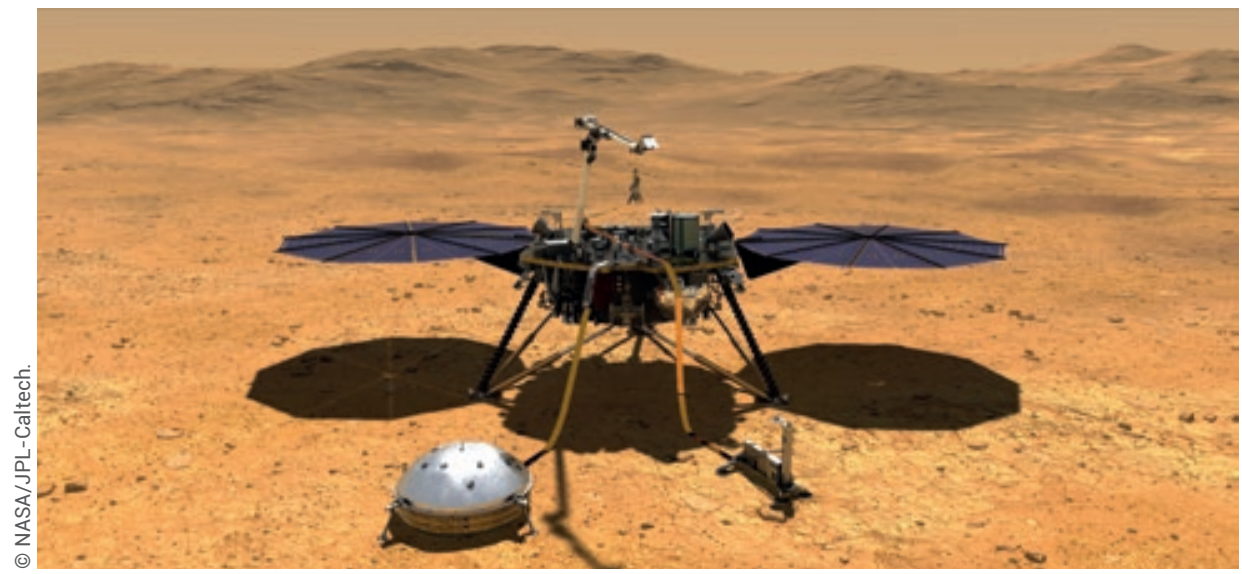

Vue d'artiste de la sonde spatiale InSight de la NASA à la surface de la planète Mars, où elle s'est posée le 26 novembre 2018. Le sismomètre SEIS (en avant-plan à gauche), conçu par l'Institut de Physique du Globe de Paris et supervisé par le CNES, est destiné à l'étude de la structure interne de Mars.
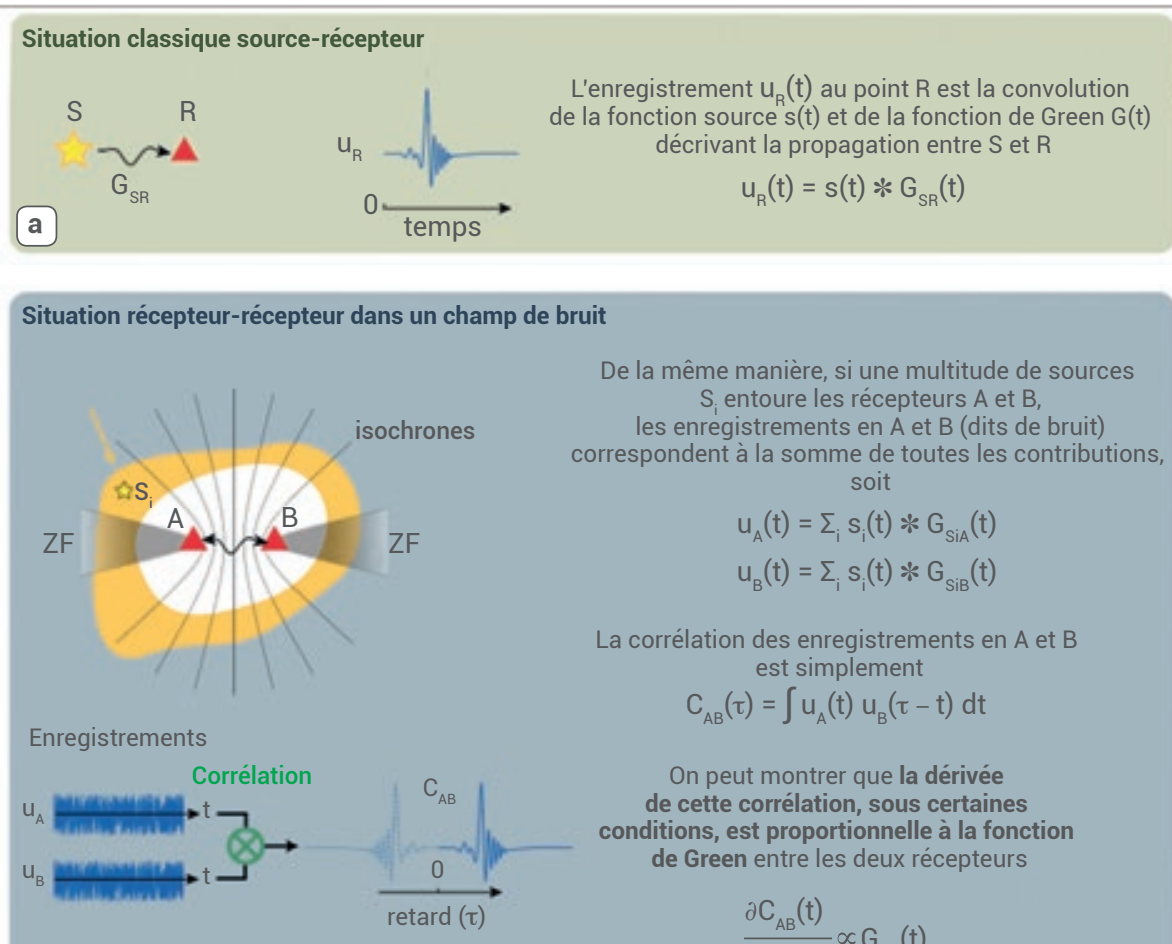

b

1. Principe de la détermination de la fonction de transfert élastodynamique d'un milieu par enregistrement des ondes sismiques.

(a) Méthode utilisant l'enregistrement de l'onde émise par une source (plus ou moins) ponctuelle. Le signal $u_{R}(t)$ (en bleu) enregistré par le récepteur $R$ correspond à la combinaison du signal de la source $S$ et de la propagation entre les deux positions, décrite par la fonction de Green $G_{S R}(t)$.

(b) Méthode utilisant les enregistrements par deux récepteurs (A et B) d'un champ d'ondes émis par une multitude de sources (symbolisées par la surface jaune), simulant le bruit sismique. Ces enregistrements continus sont intercorrélés pour reconstruire un signal proportionnel à la fonction de Green élastodynamique $\mathrm{G}_{\mathrm{AB}}(\mathrm{t})$ entre les deux récepteurs. Sur l'image en haut à gauche, les hyperboles noires symbolisent les positions où les sources interfèrent à des temps constants (isochrones). Les zones grises représentent les positions où les sources de bruit interfèrent de manière cohérente (zones de Fresnel, ZF) pour que la corrélation puisse tendre vers la réponse du milieu entre $A$ et $B$. Pour que la corrélation des enregistrements en $A$ et $B$ soit proportionnelle à la fonction de Green $G_{A B}$, il faut que le bruit sismique contienne toutes les ondes et trajets possibles dans le milieu (condition d'équipartition du champ). source réelle comme ponctuelle. Si cette simplification n'est pas justifiée, les propriétés de la source doivent être estimées au mieux pour corriger les signaux.

Une approche radicalement différente a été proposée en acoustique, puis appliquée en sismologie dès le début des années 2000. Plutôt que d'utiliser les enregistrements issus d'une source plus ou moins ponctuelle comme un séisme, l'idée est de reconstruire directement la fonction de Green entre deux récepteurs. Il faut pour cela que ces derniers enregistrent un champ d'onde complexe a priori chaotique. Un schéma conceptuel de la méthode est présenté en figure $1 \mathrm{~b}$.

En 2001, une première expérience a démontré dans le domaine ultrasonore que la corrélation d'un bruit diffus enregistré par deux récepteurs placés à la surface d'un bloc d'aluminium, converge vers la fonction de Green entre les deux récepteurs [2]. Dans cette expérience, le champ diffus (équivalent ici à la coexistence de tous les modes élastiques) est obtenu par réverbérations multiples sur les parois du volume. La qualité de la convergence vers la fonction de Green augmente alors avec la durée du signal effectivement corrélé. En d'autres termes, l'opération de corrélation (ou interférométrie) entre deux enregistrements synchrones et distants permet de reconstruire un signal déterministe simple correspondant à l'enregistrement par l'un des deux récepteurs du champ d'onde émis par une source ponctuelle impulsionnelle positionnée sur l'autre récepteur. Tout se passe comme si le champ initial comprenait 
$>>$

toutes les ondes et trajets possibles dans la structure et dans les bonnes proportions (on parle alors d'équipartition du champ d'onde), et que l'opération de corrélation permettait d'extraire (de filtrer) uniquement l'information correspondant à la propagation entre les deux récepteurs. On reconstitue ainsi la fonction de Green entre les deux points de mesure.

L'idée de reconstruire une information déterministe utilisable pour l'imagerie à partir de signaux chaotiques et dont les sources sont modélisables par des processus aléatoires, avait déjà été abordée en sismologie, principalement de manière théorique, dès la fin des années 1950. L'héliosismologie a également exploré et appliqué au Soleil, avec succès, ce type d'approche dans les années 1990.

Une expérience similaire à celle du bloc d'aluminium décrite plus haut est présentée en figure 2a. Un champ complexe d'ondes élastiques est généré à partir d'un jet d'air comprimé à la surface d'une plaque d'aluminium pendant quelques secondes. Une ligne de récepteurs enregistre le signal sur un temps long. La figure $2 \mathrm{c}$ montre le résultat de la corrélation de l'enregistrement mesuré par un récepteur de référence en extrémité de ligne $\left(\mathrm{r}_{0}\right)$ avec ceux mesurés par tous les autres récepteurs. Des ondes (dites de Lamb) directes et réverbérées sont clairement visibles à la surface de la plaque. Après corrélation, tout se passe comme si une source avait été déclenchée à la position du récepteur de référence $r_{0}$. Dans cette représentation en temps et distance, on peut par exemple mesurer très facilement la vitesse (et la dispersion) de l'onde dans la plaque. Ce résultat est transposable à la surface de la Terre, comme expliqué dans la section suivante. On peut noter que plusieurs réalisations de l'expérience (excitation de la plaque, corrélation des enregistrements) doivent être moyennées pour atteindre un résultat exploitable.

\section{La corrélation de bruit en sismologie : des applications devenues standard}

Pour transposer à la Terre l'expérience du bloc d'aluminium de Lobkis et Weaver, Campillo et Paul [3] ont d'abord montré que les ondes de surface qui forment l'essentiel de la fonction de Green entre des sismomètres distants de plusieurs dizaines de kilomètres peuvent être reconstruites par corrélation de la coda, train d'ondes diffuses visible après la partie principale (balistique) des signaux émis par un séisme, et dont l'énergie décroit rapidement avec le temps. Une étape importante a été franchie lorsque ce même résultat a été obtenu en utilisant non pas la coda des enregistrements de séismes, mais les vibrations naturelles et perpétuelles de la surface du sol qui forment le bruit sismique ambiant. Alors qu'il avait toujours été considéré comme nuisible pour la détection et l'étude des séismes, le bruit sismique ambiant est devenu, grâce à la corrélation,

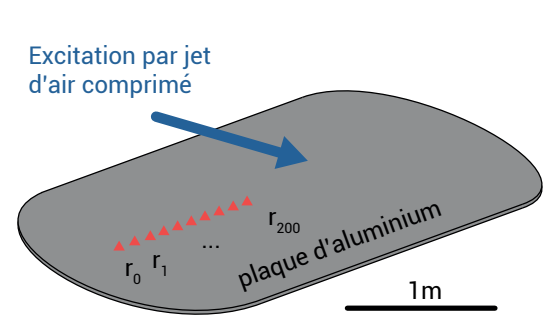

a

b
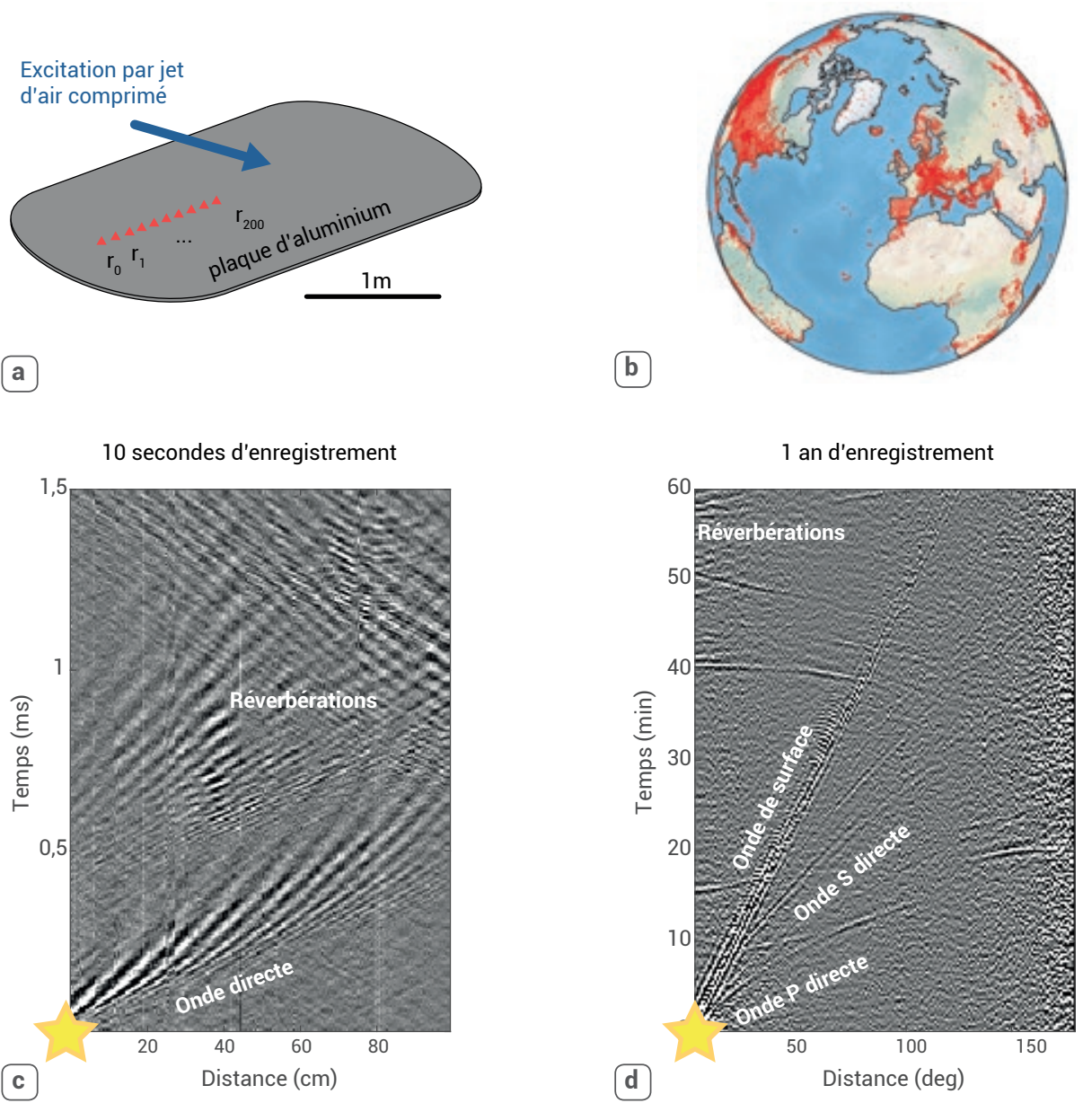

2. Expériences réelles de corrélation du champ d'ondes ambiant : (a et c) à l'échelle du laboratoire, (b et d) à l'échelle de la Terre.

(a) Génération et détection d'ondes élastiques à la surface d'une plaque d'aluminium ; les capteurs sont représentés par des triangles rouges.

(b) Distribution des stations sismiques (points rouges) à la surface de la Terre.

(c et d) Variations temporelles des fonctions de corrélation, classées selon la distance à un récepteur de référence ( $r_{0}$ dans le cas de la plaque). L'amplitude est représentée en niveaux de gris. L'axe du temps correspond à l'axe du retard dans la figure 1. Dans les deux cas, la fonction de corrélation converge vers la réponse élastodynamique du milieu (une plaque d'aluminium ou la Terre).

Sources : (c) Ludovic Moreau. (d) Modifié de Boué et al. [6]. 
pondent respectivement à la fréquence dominante de la houle et à l'interaction non linéaire de deux trains de houle se propageant en sens opposés, interaction qui génère des variations de pression sur le plancher océanique. À plus basses fréquences (en dessous de $0,01 \mathrm{~Hz}$ ), les mécanismes à l'origine du bruit sismique sont toujours débattus mais semblent principalement liés à des forçages atmosphériques et océaniques. Par chance, la bande de fréquence des deux pics microsismiques correspond précisément aux besoins de l'imagerie par ondes de surface pour les structures de la croute terrestre à l'échelle régionale (quelques centaines de kilomètres). Pour ce type de besoin, l'utilisation de sources conventionnelles (séismes, explosions) est aujourd'hui quasiment éclipsée par celle du bruit sismique ambiant qui, par interférométrie de longues séries temporelles, transforme virtuellement chaque récepteur en une source fictive. Le développement de grands réseaux d'observation sismologique constitués de plusieurs centaines de sismomètres enregistrant en continu sur des temps longs (plusieurs mois à années) a permis la progression rapide de l'imagerie par corrélation de bruit sismique, en remplacement des techniques basées sur les sources conventionnelles. En s'appuyant sur l'analyse des ondes de surface, l'imagerie sismique des structures de la croute et du manteau supérieur est devenue possible à l'échelle de continents entiers et avec une résolution jamais atteinte auparavant, y compris en l'absence totale de séismes. La démarche standard de ce type d'imagerie est la construction d'un modèle de vitesse des ondes $\mathrm{S}$ par inversion des mesures de dispersion des ondes de surface reconstruites à partir du bruit sismique ambiant. La figure 3 présente une carte de vitesse des ondes $\mathrm{S}$ à $30 \mathrm{~km}$ de profondeur sous l'Europe de l'Ouest, issue de ce type d'analyse. Pour atteindre ce niveau de résolution sur une zone aussi étendue, il a fallu intercorréler plusieurs années de bruit sismique enregistré par près de 1300 stations sismologiques.

Un second atout majeur de la méthode de corrélation de bruit sismique est sa répétabilité dans le temps. Surveiller les variations temporelles de vitesse des ondes sismiques associées à la dynamique de structures géologiques comme des failles ou des volcans était quasiment impossible faute de pouvoir éclairer ces structures par des sources identiques et répétées dans le

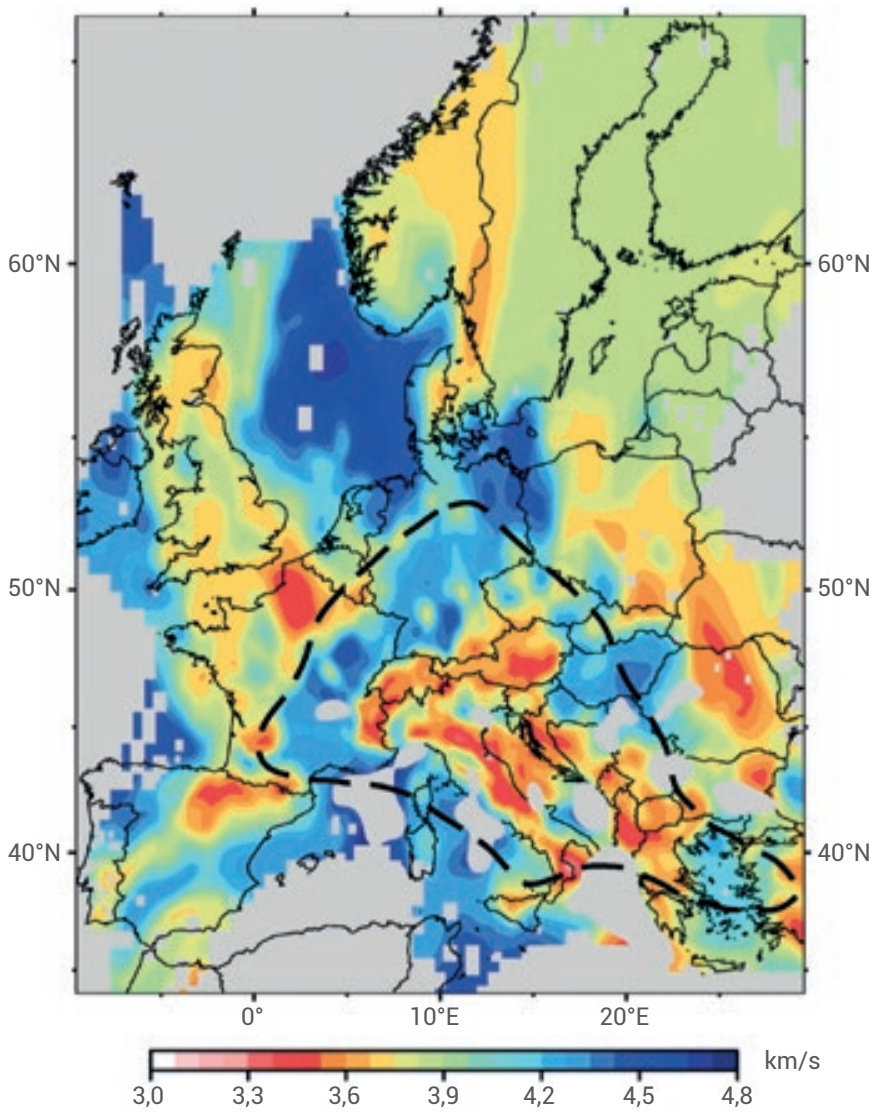

3. Carte de vitesse des ondes de cisaillement à $\mathbf{3 0} \mathrm{km}$ de profondeur sous l'Europe de l'Ouest. Mesures réalisées par analyse de la dispersion des ondes surfaciques de Rayleigh, reconstruites par corrélation des enregistrements continus de bruit sismique par les réseaux sismologiques européens permanents et temporaires. La région où l'image est la mieux résolue est délimitée par le trait en tirets noirs. Source : Lu et al. [7].

temps. Le bruit sismique dans la bande de fréquences microsismique (autour de $0,1 \mathrm{~Hz}$ ) étant un processus permanent et assez stationnaire (en temps et surtout en fréquence), sa corrélation donne des signaux significatifs sur des fenêtres de temps relativement courtes, de l'ordre d'une heure. Ainsi, il devient possible de sonder régulièrement le milieu et de mesurer de manière extrêmement fine son évolution. Par cette méthode et à une échelle spatiale de plusieurs kilomètres (par exemple sur un volcan ou une faille active), on peut mettre en évidence des variations relatives des vitesses des ondes sismiques de l'ordre de 0,01\% avec une résolution temporelle de l'ordre de quelques heures. Plusieurs exemples de ce type d'applications sont présentés dans la référence [5]. Le défi le plus important reste encore aujourd'hui d'observer des variations des propriétés mécaniques du milieu géologique avant l'occurrence d'un séisme, observation déjà réalisée à l'échelle du laboratoire.

\section{Des limitations et des solutions en développement}

Au-delà du succès des méthodes d'imagerie qui lui sont associées, il faut noter que la technique de corrélation du bruit sismique possède un certain nombre de limitations. La fonction de corrélation ne converge que très partiellement vers la véritable fonction de Green élastodynamique du milieu, puisque ce sont majoritairement les ondes de surface qui sont reconstruites du fait de leur surreprésentation dans le champ de bruit naturel. Une conséquence directe est donc la limitation a priori de la résolution des images en profondeur, puisque la sensibilité des ondes de surface aux structures qu'elles traversent décroit rapidement avec la profondeur. La bande de fréquences accessible étant fatalement limitée à celle du bruit ambiant, elle ne permet en général que la reconstruction du mode fondamental des ondes de surface. L'amplitude des 
ondes reconstruites par corrélation de longues séries temporelles de bruit est souvent biaisée et son utilisation directe n'est que rarement possible. Ces amplitudes ont pourtant une importance cruciale pour mesurer l'atténuation du milieu ou encore caractériser les effets de site - amplifications ou atténuations par les structures locales pour l'évaluation du risque sismique. Calibrer l'amplitude des corrélations à partir d'enregistrements de séismes ou du calcul de sismogrammes synthétiques reste généralement nécessaire pour ce type d'application. Enfin, il peut arriver que le bruit sismique ne provienne que d'une seule direction, ce qui compromet même la reconstruction des ondes de surface par corrélation pour certains couples de stations.

Qu'il s'agisse de mesurer des amplitudes ou des vitesses de propagation, les biais éventuels proviennent toujours d'un défaut de convergence des corrélations vers la fonction de Green lié au non-respect de la condition d'équipartition du champ d'onde généré que constitue le bruit ambiant (équilibre d'énergie entre les ondes $\mathrm{P}$ et $\mathrm{S}$ par diffusion et isotropie du champ). Aux fréquences qui intéressent les sismologues, le problème trouve généralement son origine dans une répartition spatiale ou temporelle inadéquate des sources de bruit autour de la zone étudiée. Il est intéressant de constater que les limitations évoquées ici sont finalement très proches de celles que l'on espérait éviter en remplaçant les séismes par des corrélations de bruit : il est toujours préférable de connaitre les paramètres de la source du signal. En s'attaquant directement à l'étude de l'origine et des propriétés du bruit ambiant, une multitude de travaux récents montrent qu'il est encore possible de repousser les frontières du domaine d'utilisation des corrélations de bruit pour l'imagerie et la surveillance sismique à toutes les échelles.

À l'échelle globale, on sait depuis des dizaines d'années que le bruit sismique ambiant contient des ondes de volume qui se propagent à travers toute la planète et trouvent leur source dans les grandes tempêtes océaniques. La corrélation de bruit entre deux récepteurs placés à la surface de la Terre et distants de plusieurs milliers de kilomètres permet-elle de reconstruire ces ondes de volume et d'accéder ainsi à de nouvelles informations sur la structure profonde de la Terre ? La réponse positive à cette question a largement bénéficié de l'implantation récente de réseaux denses de plusieurs centaines à milliers de sismomètres de haute qualité fonctionnant pendant plusieurs années en continu, et de la distribution libre de leurs données. Le résultat est une augmentation significative de la qualité des enregistrements, permettant l'émergence dans les corrélations de bruit d'une multitude de trajets habituellement visibles seulement dans les enregistrements de forts séismes. La figure $2 \mathrm{~d}$ montre les signaux obtenus par corrélation d'une année de bruit enregistré par des stations sismologiques réparties à la surface de la Terre (fig. 2b). Les corrélations sont ici classées en fonction de la distance (en degrés) qui sépare les couples de stations. En plus de l'onde de surface, on distingue nettement les ondes directes $\mathrm{P}$ et $\mathrm{S}$ ayant traversé le manteau. Les arrivées tardives, à partir de $40 \mathrm{mn}$, correspondent à des ondes réverbérées à l'antipode et ayant transité à travers le manteau et le noyau.

L'utilisation d'un grand nombre de stations réparties à la surface de la Terre (fig. 2b) met en lumière la structure moyenne, radiale, de la Terre. Pour aller au-delà et caractériser des hétérogénéités de structure, ce type de reconstruction doit rester possible pour des trajets, et donc des couples de stations précis correspondant à des signaux extrêmement ténus. Afin d'optimiser les capacités d'observation de ces signaux faibles, on regroupe des stations sismologiques en antennes (ou miniréseaux) pour abaisser le seuil de détection. Différentes stratégies de filtrage permettent aussi de réduire les signaux parasites. Plutôt que de corréler de longues séries de bruit ambiant, une stratégie alternative pour reconstruire ces ondes de volume est de corréler des séries temporelles plus courtes, mais bien situées dans la coda (signaux tardifs) d'enregistrements de forts séismes. Ceux-ci génèrent en effet un champ riche en ondes de volume qui se réverbèrent à l'intérieur de la Terre pendant plusieurs jours après l'évènement. Des travaux récents discutent les avantages et inconvénients de l'utilisation de la coda des séismes plutôt que du bruit sismique pour sonder la Terre profonde. Dans les deux cas, la possibilité de réaliser des mesures locales par corrélations entre deux stations est une approche très prometteuse, alors que l'imagerie de la Terre profonde à partir des séismes est très limitée par leur distribution hétérogène à la surface du globe. On peut notamment citer la résolution limitée des modèles d'anisotropie de vitesse sismique de la graine (noyau interne), simplement due à l'absence de séismes en zones polaires (Arctique et Antarctique).

À plus petite échelle, des travaux récents permettant d'améliorer la convergence de la corrélation de bruit vers la fonction de Green ouvrent des perspectives importantes pour la surveillance à haute résolution en temps et en espace de structures géologiques actives. On retrouve notamment ce type d'applications de surveillance « passive " dans des domaines de recherche aujourd'hui très dynamiques, comme les nombreux travaux sur le rôle des fluides dans l'initiation des séismes. Il s'agit par exemple de mieux comprendre les mécanismes responsables de la sismicité à proximité de certaines zones d'exploitation d'hydrocarbures (sismicité induite).

Enfin, les méthodes présentées dans cet article trouvent une résonance particulière aujourd'hui, alors qu'un sismomètre vient d'être déployé sur Mars par la mission spatiale InSight (voir la photo, p. 13). L'utilisation du bruit de fond et plus généralement de la corrélation de champs complexes, ou de l'autocorrélation dans le cas de l'unique station InSight, pourrait se révéler déterminante dans la caractérisation de la structure interne d'autres corps du système solaire, pour certains dépourvus de tectonique active et donc de séismes.

\section{Références}

1 B. Romanowicz, Reflets de la physique 56 (2018) 4-9.

2• O.I. Lobkis et R.L. Weaver, J. Acoust. Soc. Am., 110 (2001) 3011-3017.

3. M. Campillo et A. Paul, Science, 299 (2003) 547-549.

4- N.M. Shapiro et M. Campillo, Geophys. Res. Lett., 31 (2004) L07614.

5• N. Nakata, L. Gualtiéri et A. Fichtner (eds.), Seismic ambient noise,

Cambridge University Press (2019).

6. P. Boué et al., Geophys. J. Int., 194 (2013) 844-848.

7• Y. Lu et al., Geophys. J. Int., 214 (2018) 1136-1150. 